\title{
Sarcoidosis and immunoglobulin lambda II light-chain amyloidosis diagnosed after orthotopic heart transplantation: a case report and review of the literature
}

\author{
Diana O Treaba ${ }^{1}$, Merrill D Benson ${ }^{2}$, Lina W Assad ${ }^{1}$ and John R Dainauskas ${ }^{1}$ \\ ${ }^{1}$ Rush Presbyterian St Luke's Medical Center, Chicago, IL, USA and ${ }^{2}$ Department of Pathology and Laboratory \\ Medicine, Indiana University School of Medicine, Indianapolis, IN, USA
}

\begin{abstract}
Cardiac involvement by sarcoidosis and concomitant deposition of $\mathrm{AL}$ amyloid is an uncommon association. We describe the case of a 53-year-old African-American man with a 7-year history of dilated nonischemic cardiomyopathy and severe cardiac failure who underwent orthotopic heart transplantation. His prior cardiac biopsies had only mild myocyte hypertrophy and minimal interstitial fibrosis. After surgery, numerous sarcoid granulomas and amyloid deposition were identified in the native heart. Six days after the transplant the patient died due to aspiration bronchopneumonia and acute renal failure. At autopsy, both sarcoidosis and immunoglobulin (Ig) lambda light-chain amyloidosis were present in the native atria, lungs, thyroid, liver, spleen, and kidneys. Sarcoid granulomas alone were present in the parathyroids, lymph nodes, and bone marrow. Amyloid deposition alone was present in the aorta, stomach, large bowel, and urinary bladder. There was no evidence of plasma cell dyscrasia, or underlying gammopathy. This unusual association was described in only two other cases in the medical literature. However, this is the first case of sarcoidosis and AL amyloidosis with successful sequencing and identification of lg lambda light-chain amyloid, and in which there was no evidence of plasma cell dyscrasia.
\end{abstract}

Modern Pathology (2005) 18, 451-455. doi:10.1038/modpathol.3800277

Keywords: sarcoid granulomas; lambda light chain; amyloidosis

Sarcoidosis is a multisystem disorder characterized by an increased cellular immune response at sites of disease activity. The disease has a higher incidence in the African-American population and may be initiated by exposure to yet unknown environmental agents. ${ }^{1}$ Cardiac involvement in sarcoidosis is clinically reported in $1-6 \%$ of the patients, ${ }^{2}$ while myocardial granulomas have been found in 15-25\% of autopsies. ${ }^{3}$ The diagnosis of cardiac sarcoidosis can be definitively made by endomyocardial biopsy. Although this test can demonstrate the presence of noncaseating granulomas, it has a low diagnostic rate due to the patchy distribution of the disease. ${ }^{4}$

The association of cardiac sarcoidosis and amyloidosis is reported in only seven cases in the English literature of the last 30 years. ${ }^{5}$ Five case reports describe the association of cardiac sarcoido-

Correspondence: Dr DO Treaba, MD, PhenoPath Laboratories, 551 North 34th Street, \#100, Seattle, WA 98103, USA.

E-mail: dtreaba@msn.com

Received 13 April 2003; revised 21 April 2004; accepted 26 June 2004 sis and AA amyloidosis, ${ }^{1}$ while the remaining two case reports emphasize the association with AL amyloidosis. The first of these two last case reports ${ }^{5}$ suggests that the association between sarcoidosis and $\mathrm{AL}$ amyloidosis was coincidental, due to coexistence of a multiple myeloma. The second report $^{6}$ explains the association as a possible consequence of AL amyloid production by an increased plasma cell population present in the bone marrow.

We present the first report of cardiac involvement by sarcoidosis and AL amyloidosis, with identification of immunoglobulin lambda class II light-chain deposition, and no evidence of a concurrent plasma cell dyscrasia.

\section{Case report}

The patient was a 53-year-old African-American man with a 7-year history of nonischemic dilated cardiomyopathy, diagnosed in 1995, hypertension, hypercholesterolemia, and osteoarthritis. A first cardiac biopsy, obtained in October 1995, had mild 
Table 1 Tryptic peptides isolated from cardiac amyloid fibrils

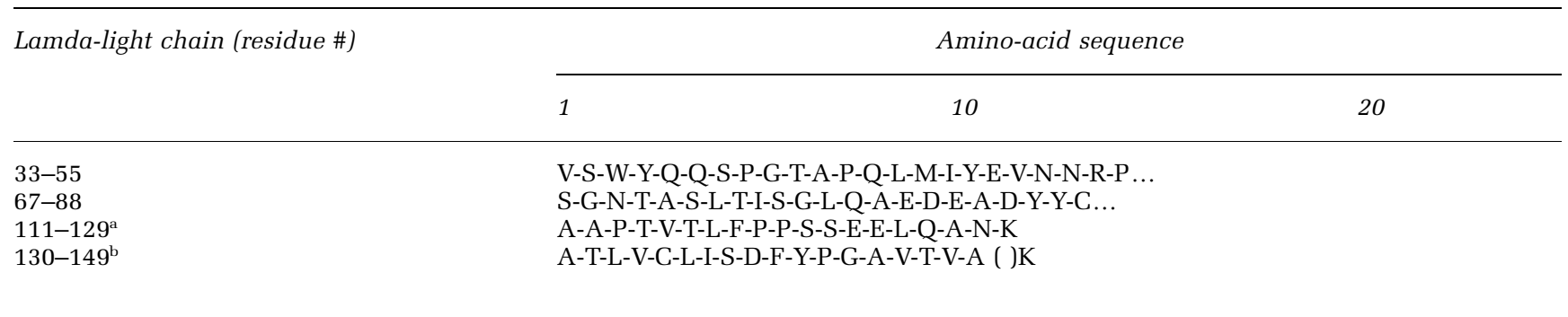

${ }^{\mathrm{a}}$ The lambda constant region is MCG (+) with Thr114.

${ }^{\mathrm{b}}$ No amino acid was identified at residue 148 which is usually Trp.

myocardial hypertrophy and minimal interstitial fibrosis. In the same month, an HLA immunophenotyping showed positivity for several HLA class I and II antigens: A28, B42, B53, Cw2, Cw4, Bw4, Bw6, DR11, DR18, DQ1, DQ4, and DR52. During the next 4 years, the patient developed chronic heart failure, third-degree atrio - ventricular block, and several episodes of ventricular fibrillation for which in March 1999 he received an automatic implantable cardioverter defibrillator. This was replaced in January 2001 with a left ventricle assist device. The left ventricle core biopsy obtained during the placement of the left ventricle assist device also had only myocyte hypertrophy and minimal interstitial fibrosis. During the following year, the patient had multiple drive line infections, increased left and right ventricular pressures, and marked fatigue. In March 2002, he underwent an orthotopic heart transplantation. Immediately after surgery, the patient developed persistent tachycardia, and due to severe shortness of breath was intubated. His laboratory tests showed a white blood cell count of $21.7 \times 10^{9} / \mathrm{l}$, a red blood cell count of $4.11 \times 10^{12} / \mathrm{l}$, hemoglobin $10.3 \mathrm{~g} / \mathrm{dl}$, platelets $142 \times 10^{9} / \mathrm{l}$, serum urea nitrogen $28 \mathrm{mg} / \mathrm{dl}$, and creatinine level of $2.4 \mathrm{mg} / \mathrm{dl}$. He underwent dialysis in the next days after surgery due to progressive increase of serum urea nitrogen up to $118 \mathrm{mg} / \mathrm{dl}$ and creatinine levels up to $7.4 \mathrm{mg} / \mathrm{dl}$, associated with high levels of SGOT $16224 \mathrm{IU} / \mathrm{l}$, SGPT $6251 \mathrm{IU} / \mathrm{l}$, and LDH up $76416 \mathrm{IU} /$ l. On postoperative day 6 , the patient suffered an episode of coffee-ground emesis, and an abdominal X-ray showed dilated small and large bowel. His blood pressure and cardiac output could not be stabilized and he died a few hours later. An autopsy, without examination of the brain, was performed.

\section{Materials and methods}

\section{Histology/Immunochemistry}

Examination of the native heart, and autopsied organs was performed. The tissue samples were fixed in formalin $(10 \%)$ and embedded in paraffin. Paraffin sections were routinely stained with hematoxylin and eosin. GMS, FITES, PAS, and acid-fast stains were performed to exclude a possible infectious etiology of the granulomas.

In order to characterize the deposited amyloid, Congo red staining was performed according to the method of Puchtler. ${ }^{7}$ Pretreatment with potassium permanganate before Congo red staining was also performed in order to evaluate the resistance of birefringence to oxidation. This method characterizes AL amyloid deposition if the birefringence persists, while AA-type amyloid birefringence disappears after oxidation. Fluorescence of the deposited amyloid was also studied with Thioflavine $\mathrm{T}$ dye method, less specific for amyloid detection than Congo red.

Cocktails of oligonucleotide probes labeled with fluorescein were used to detect cytoplasmic immunoglobulins lambda and kappa (Ventana, INFORM, Catalog \# 780-2843 and 780-2844) for examination of the bone marrow.

Immunohistochemistry for AA (Novocastra, UK), transthyrethin (TTR) (DAKO, Denmark), and cytoplasmic kappa and lambda immunoglobulins light chains was performed on paraffin-embedded sections. Amyloid fibrils were isolated from paraffinembedded tissue from the native heart, solubilized in $6 \mathrm{M}$ guanidine, reduced and alkylated, and digested with trypsin.

Tryptic peptides were separated by high-performance liquid chromatography, and sequenced in an ABI 773A (491 abc) protein sequencer (Table 1).

Electron microscopy studies of amyloid deposition were performed on tissues obtained from a paraffin-embedded section of the native heart.

\section{Results}

Multiple sections of the native heart contained small well-demarcated noncaseating single or clustered granulomas, in both ventricles, especially in their basal regions, and also in the upper region of the interventricular septum. Granulomas were present within the epicardium, myocardium (Figure 1), endocardium, and in a crescentic distribution in the adventitia of intramural and epicardial coronary arteries. The mitral valve had extensive fibrosis and 


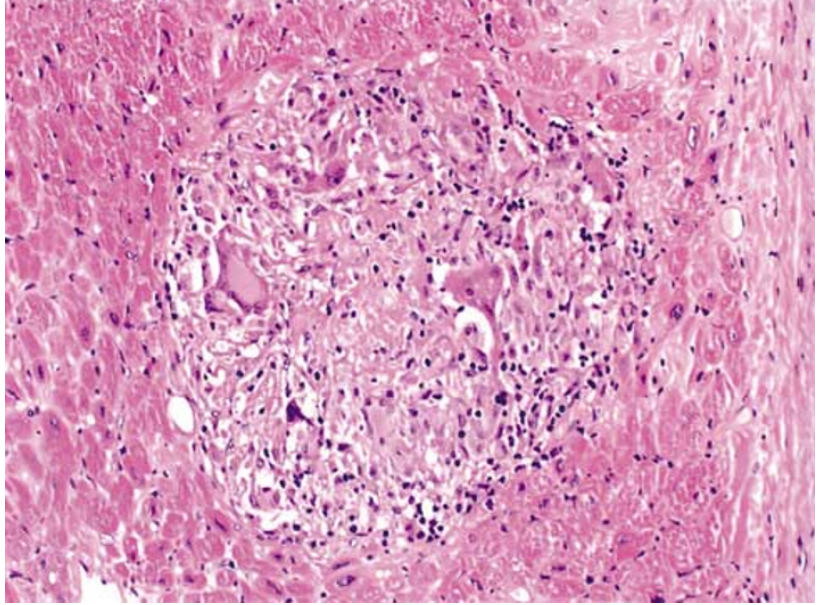

Figure 1 Sarcoid granulomas located in the myocardium, hematoxylin and eosin, $\times 100$.

clusters of small granulomas, predominantly in the base of the valve. Noncaseating granulomas were present in the left native atrium, in both lungs, involving the blood vessel walls and the interalveolar septa, subpleural, and in the spleen, where large areas of fibrosis and clusters of granulomas had completely distorted the normal parenchyma. Multiple granulomas were also seen in the liver, pancreas, thyroid, parathyroids, kidneys, mediastinal and abdominal lymph nodes, and bone marrow. The granulomas were present in different stages of evolution. The majority were composed of epithelioid histiocytes and multinucleated giant cells, which occasionally showed eosinophilic asteroid bodies in their cytoplasm. Some older granulomas were surrounded by fibrosis, or were almost completely replaced by fibrosis. All special stains for organisms were negative, supporting a diagnosis of sarcoidosis. There was extensive deposition of pale eosinophilic material in the native heart, distributed as large nodules or around individual myocytes in both atria and ventricles, and in the walls of intramyocardial blood vessels, sometimes in close proximity to the granulomas. With Congo red stain this eosinophilic material had the apple-green birefringency characteristic of amyloid (Figure 2). In slides pretreated with potassium permanganate, the apple-green birefringency remained unchanged suggesting deposition of AL amyloid. Thioflavin T highlighted the presence of amyloid within the myocardial interstitium and in the blood vessel walls. The deposited amyloid-stained negative with AA-antisera, and slightly positive with AL-antisera, while the sequencing of amyloid revealed deposition of Ig lambda II light chain. Amyloid deposition was also noted in the aortic wall, interalveolar septa and in the walls of the pulmonary vessels, splenic parenchyma, walls of the stomach, large bowel, urinary bladder, and in the glomerular mesangium.

Electron microscopy studies of a paraffin-embedded section of the right ventricle showed

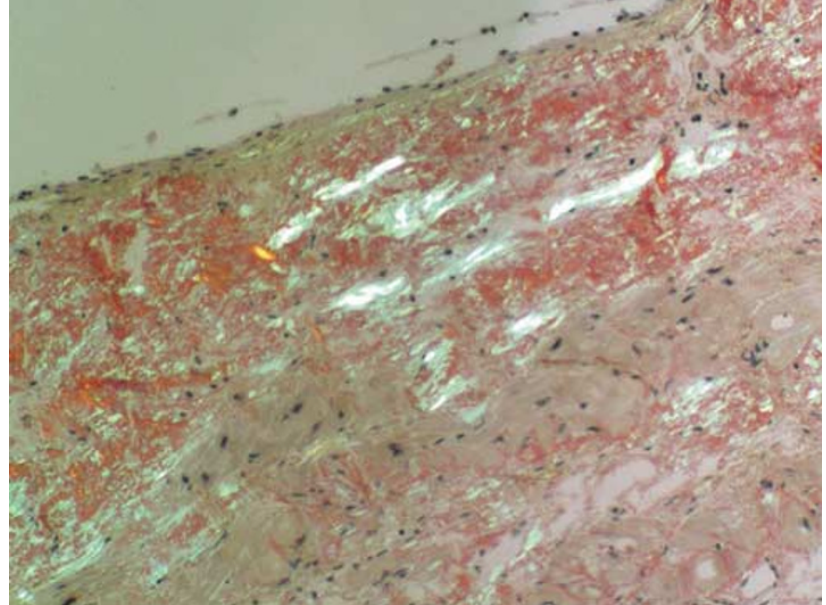

Figure 2 Subepicardial deposition of amyloid, Congo red stain, $\times 200$.

deposition within the myocardial interstitium of randomly arranged nonbranching, slender fibrils of amyloid.

The bone marrow biopsy and aspirate had no increase in the plasma cell population that was polyclonal with an approximately $2: 1$ kappa to lambda ratio.

The transplanted heart had mild myocardial hypertrophy, and focal microscopic areas of necrosis at the anastomotic line, but no sarcoidosis or amyloidosis.

\section{Discussion}

Since 1877, when Sir Jonathan Hutchinson described sarcoid skin lesions, progress was made in the diagnosis and treatment of the disease. However, the disease etiopathogenesis and its clinical course are still less understood. The most frequently involved organs are the lungs and the hilar lymph nodes-affected in up to $90 \%$ of patients. The skin, eyes, and peripheral lymph nodes are involved in $20-30 \%$ of patients. The spleen, liver, bone, kidneys, or central nervous system may be involved in $2-6 \%$ of patients. ${ }^{8}$ The heart is involved in $1.1 \%{ }^{9}$ up to $6 \%$ of the patients. ${ }^{8}$

Cardiac involvement by sarcoidosis has a patchy distribution with granulomas present especially in the proximal portion of the left ventricle, upper interventricular septum, and rarely within the atria. ${ }^{10}$ This patchy distribution may explain the low diagnostic rate of sarcoidosis on endomyocardial biopsies $11.2^{11}-35.5 \% .^{12}$

Cardiac sarcoidosis is symptomatic in only $5 \%$ of all patients, ${ }^{2}$ may manifest with arrhythmias, heart blocks, cardiac failure ${ }^{13}$ and may lead to heart transplantation. ${ }^{14}$

Sarcoidosis has been reported in association with different sets of histocompatibility antigens. HLA A1, B8, and DR3 have been associated with familial clustering, ${ }^{15}$ HLA DR17, ${ }^{15}$ DR52 ${ }^{16}$ are associated 
with a limited disease and usually a good outcome, while HLA DR15 and DR16 are associated with systemic disease. ${ }^{17}$ The HLA immunophenotyping in our patient showed among other HLA antigens, positivity for HLA DR52; however, the evolution of his disease was more severe than expected based only on the presence of this HLA antigen.

The association between sarcoidosis and amyloidosis is rarely reported. Several types of amyloid proteins can be deposited in the heart. The most frequently reported are $\mathrm{AA}, \mathrm{AL}$, and transthyretin and only rarely apolipoprotein A-I. ${ }^{18}$ Only AA- and AL-type amyloid have been reported in association with cardiac sarcoidosis. In AA amyloidosis, there is usually minimal deposition of amyloid within the myocardial interstitium and walls of small arterioles. As a long-standing inflammatory process sarcoidosis leads to increased concentrations of an acute phase reactant-serum amyloid A. Serum amyloid A is actually a family of apolipoproteins, which in active sarcoidosis can increase to levels 1000-fold higher than those found in the noninflammatory diseases. SAA is also the precursor protein of AA amyloid. Salazar et $a l^{1}$ observed that several inflammatory diseases including sarcoidosis are associated with hypocholesterolemia, especially with low HDL-cholesterol levels. This pattern of depressed HDL-cholesterol is seen in active and untreated sarcoidosis and may explain why these patients are prone to develop coronary artery disease.

AL amyloidosis is associated in the majority of cases with some type of plasma cell dyscrasias; however, in about $14 \%$ of the cases an underlying gammopathy could not be determined. ${ }^{19}$ The proliferation of B lymphocytes, possibly secondary to repeated exposure to an infectious antigen, is followed by hyperproduction of immunoglobulin light chains. They are later on deposited and polymerized in the tissues to form AL amyloid. There is usually extensive deposition of AL amyloid in the myocardial interstitium (in a nodular, perimyocytic, or mixed distribution), intramural coronary arteries, endocardium, pericardium, and atrioventricular valves. ${ }^{20}$

The most likely cause of amyloidosis in our patient appears to be his undiagnosed active sarcoidosis. Significant for his case is the absence of amyloid or granulomas in the prior myocardial biopsies from 1995 or 2001. The low diagnostic rate of sarcoidosis on endomyocardial biopsy ${ }^{4}$ can be explained by the location of the sarcoid granulomas, especially in the base of the heart, while the biopsy is usually taken from the apex of the right ventricle. A similar explanation is valid for the negative core biopsy obtained in our patient. During the placement of an LVAD, the core biopsy is usually taken from the apex of the left ventricle a region rarely affected by sarcoidosis.

Both biopsies were also negative for amyloid. It is however possible that amyloidosis had developed during the 1 year interval between the placement of the LVAD, when the core biopsy was taken, and the orthotopic heart transplantation. This may have been possible in the background of an active systemic sarcoidosis with superimposed multiple drive line infections. However, in this scenario, AA amyloid deposition is most likely expected. In animal models of amyloidosis, in mice given inflammatory stimuli, AA amyloid had developed after 3-6 weeks. It has also been postulated that the deposited amyloid has a dynamic turnover, with a possibility of regression if the source of amyloid precursors is suppressed. ${ }^{19}$

The relationship between cardiac AL amyloidosis and active sarcoidosis is yet less understood. Two prior reports suggest a coincidental association between cardiac sarcoidosis and AL amyloidosis, due to concurrent presence of a plasma cell dyscrasia. $^{6,7}$ In our case, however, no plasma cell dyscrasia was present, raising the possibility of a direct relationship between active untreated sarcoidosis and development of AL amyloidosis. A possibility, which should be considered, is the appearance of a monoclonal lymphocytic population in the background of sarcoidosis. And if so, is this population responsible for production of Ig light chains, which will be later on deposited as AL amyloid? There are case reports of associations between sarcoidosis, sarcoid-like granulomas, and Hodgkin's and non-Hodgkin's lymphomas. ${ }^{21} \mathrm{~A}$ sarcoidosis-lymphoma syndrome, with a median interval of 2 years between the diagnosis of sarcoidosis and the onset of the lymphoproliferative disorder was also described. ${ }^{22}$ In our case, however, there was no evidence of a lymphoproliferative disorder.

Treatment with heart transplantation for cardiac sarcoidosis is rare, being reported only in seven cases, in three of them the diagnosis of sarcoidosis being made only after the transplantation, as in our case, after the histopathologic examination of the native heart. ${ }^{14}$

Usually, a diagnosis of cardiac amyloidosis carries a poor prognosis, with a median survival of less than 5 months in AL amyloidosis, and 2-5 years in AA amyloidosis. $^{23,24}$ The treatment with orthotopic heart transplant in amyloidosis, or sarcoidosis does not appear to stop the progression of the disease $;^{5}$ in fact, both sarcoidosis and amyloidosis may be expected to recur in transplanted hearts.

\section{References}

1 Salazar A, Pinto X, Mana J. Serum amyloid A and highdensity lipoprotein cholesterol: serum markers of inflammation in sarcoidosis and other systemic disorders. Eur J Clin Invest 2001;31:1070-1077.

2 Hagemann GJ, Wurm K. The clinical, electrocardiographic and pathololgic features of cardiac sarcoidosis. In: Jones WW, Davies BD (eds). Sarcoidosis and Other Granulomatous Diseases, 8th International Conference. Alpha Omega: Cardiff, 1980, p 601. 
3 Matsui Y, Iwai K, Tachicana T, et al. Clinico-pathological study on a fatal myocardial sarcoidosis. Ann NY Acad Sci 1976;278:445-469.

4 Uemura A, Morimoto S, Hiramitsu S, et al. Histologic diagnostic rate of cardiac sarcoidosis: evaluation of endomyocardial biopsies. Am Heart J 1999;138(Part 1): 299-302.

5 Stather D, Ford S, Kisilevsky R. Sarcoid, amyloid and acute myocardial failure. Mod Pathol 1998;11: 901-904.

6 Rakhit RD, Cleshman GJ, Cleland JGF. Sarcoid, amyloid and heart failure. Int J Cardiol 1993;41:180-182.

7 Churukian CJ. Improved Puchtler's Congo red method for demonstrating amyloid. J Histotechnol 2000;23: 139-141.

8 Lynch III JP, Sharma OP, Baughman RP. Extrapulmonary sarcoidosis. Semin Respir Infect 1998;13: 229-254.

9 Felker GM, Weimin Hu, Hare J, et al. The spectrum of dilated cardiomyopathy: The Johns Hopkins Experience with 1,278 patients. Medicine 1999;78: 270-283.

10 Mitchell DN, du Bois RM, Oldershaw PJ. Cardiac sarcoidosis: a potentially fatal condition that needs expert assessment. BMJ 1997;314:320-321.

11 Yoshida Y, Morimoto S, Hiramitsu S, et al. Incidence of cardiac sarcoidosis in Japanese patients with highdegree atrioventricular block. Am Heart J 1997;134: 362-366.

12 Fleming HA. Death from sarcoid heart disease. United Kingdom series 1971-1986. 300 cases with 138 deaths. In: Grassi CG, Pozzi E (eds). Sarcoidosis and Other Granulomatous Disorders, 11th World Congress. Elsevier: Amsterdam, 1998, p 19.

13 Roberts WC, McAllister HA, Ferrans VJ. Sarcoidosis of the heart. A clinicopathologic study of 35 necropsy patients (group I) and review of 78 previously described necropsy patients (group II). Am J Med 1977;63:86.
14 Donsky AS, Escobar J, Capehart J, et al. Heart transplantation for undiagnosed cardiac sarcoidosis. Am J Cardiol 2002;89:1447-1449.

15 Martinetti M, Tinelli C, Kolek V, et al. 'The sarcoidosis map': a joint survey of clinical and immunogenetic findings in two European countries. Am J Respir Crit Care Med 1995;152:557-564.

16 Kunikane H, Abe S, Yamaguchi E, et al. Analysis of restriction fragment length polymorphism for the HLADR gene in Japanese patients with sarcoidosis. Thorax 1994;49:573-576.

17 Hamidi AK, Liepnieks JJ, Nakamura M, et al. A novel apolipoprotein A-I variant Arg 173Pro, associated with cardiac and cutaneous amyloidosis. Biochem Biophys Res Commun 1999;257:584-588.

18 Berlin M, Fodgell-Hahn A, Olerup O, et al. HLA-DR predicts the prognosis in Scandinavian patients with pulmonary sarcoidosis. Am J Respir Crit Care Med 1997;156:1601-1605.

19 Gilmore JD, Hawkins PN, Pepys MB. Amyloidosis: a review of recent diagnostic and therapeutic developments. Br J Hematol 1997;99:245-256.

20 Roberts WC, Bruce WF. Cardiac amyloidosis causing cardiac dysfunction: analysis of 54 necropsy patients. Am J Cardiol 1983;52:137-146.

21 Dunphy HC, Panella MJ, Grosso EL. Low grade B-cell lymphoma and concomitant extensive sarcoid like granulomas. A case report and review of the literature. Arch Pathol Lab Med 2000;124:152-156.

22 Brinker H. The sarcoidosis-lymphoma syndrome. Br J Cancer 1986;54:467-473.

23 Gertz MA, Kyle RA. Secondary systemic amyloidosis: response and survival in 64 patients. Medicine 1991; 70:246-256.

24 Hosenpud JD, DeMarco T, Frazier OH, et al. Progression of systemic disease and reduced long-term survival in patients with cardiac amyloidosis undergoing heart transplantation. Follow-up results of a multicenter survey. Circulation 1991;84(5 Suppl):338-343. 